HStud 23 (2009)2, 223-246 DOI: 10.1556/HStud.23.2009.2.5

\title{
DIE UNGARISCHE TANZHAUS-BEWEGUNG
}

\author{
EIN BEISPIEL FÜR VOLKSKULTUR IN DER MODERNE
}

\author{
JULIA HELL \\ Technische Universität Chemnitz, Chemnitz \\ Deutschland
}

\begin{abstract}
Ein typischer Tanzhaus-Abend ist geprägt von einer ausgelassenen Stimmung unter jungen Menschen, Gemeinschaftsgefühl und aktiver Teilnahme an der Reproduktion von Volksmusik und Volkstanz. Vor allem junge Intellektuelle setzten sich aus Unzufriedenheit mit der staatlich gelenkten ,sozialistischen Folklore“ auf ihre eigene Art mit der ungarischen Volkskultur auseinander. Das ungarische Tanzhaus hat sich seit der ersten Veranstaltung im Jahre 1973 von einer teilweise politisch motivierten Bewegung zu einer Tanzhaus-Szene entwickelt, die heute ihren festen Platz in der ungarischen Unterhaltungskultur einnimmt. In der von Fortschrittsdenken und Schnelllebigkeit geprägten Moderne wird durch die Tanzhaus-Enthusiasten bewusst auf die Güter der ungarischen Volkskultur zurückgegriffen. So wird eine neuartige, experimentelle Auseinandersetzung mit traditioneller Musik und den entsprechenden Tänzen gefördert. Das ungarische Tanzhaus als Retrokultur vereint Traditionsbehaftetes mit Neuartigem und stellt demnach eine an moderne Ansprüche angepasste Form der Volkskulturpflege dar. Somit besteht die besondere Rolle der Tanzhaus-Bewegung sowie der heutigen Tanzhaus-Szene für die Volkskultur in der Moderne darin, dass bei den Veranstaltungen ein für ihr Fortbestehen notwendiges Bild vermittelt wird: Volkskultur ist nichts Vergangenes oder Vergehendes, sondern etwas Gegenwärtiges.
\end{abstract}

Schlüsselwörter: Ungarn, Sozialismus, Kulturpolitik, Tanzhaus, Volkskultur, Volksmusik, Volkstanz, Folklorismus, Retrokult

\section{Die ungarische Tanzhaus-Bewegung}

\subsection{Allgemeines}

Herkunft des Begriffs

Eine eingehende Betrachtung des Entstehungskontextes der ungarischen Tanzhaus-Bewegung ist hilfreich, um die Anliegen sowie die Entwicklung der Bewegung umfassender verstehen zu können. Der Begriff Tanzhaus - auf ungarisch táncház - hat eine doppelte Bedeutung. Einerseits verweist er offensichtlich 
auf den Ort des Geschehens und andererseits auf die beliebte Unterhaltungsform an sich, bei der zu traditioneller Volksmusik gruppen- oder auch paarweise getanzt wird.

Diese Unterhaltungsform wurde in Siebenbürgen praktiziert, daher gilt diese Region als das Ursprungsgebiet der Tanzhaus-Bewegung. Besonders die ungarische Gemeinde von Szék - heute das rumänische Sic - bot eine reiche Tradition an Volksmusik und Volkstanz und entwickelte sich zum „Mekka“ der Musiker und Tänzer sowie der Ethnologen, welche anschließend die Tanzhaus-Bewegung gründeten. Bedeutende Rollen spielten György Martin, László Lajtha und Ferenc Novák, denn sie erforschten die in Szék vorgefundene Volksmusik sowie die Volkstänze, publizierten wissenschaftliche Arbeiten und verbreiteten ihr Wissen und Können in den ungarischen Städten.

Die wöchentlichen Tanzhaus-Abende wurden von den jugendlichen, unverheirateten Bewohnern Siebenbürgens organisiert. Sie mieteten ein geeignetes Bauernhaus oder verabredeten sich für einen Abend in einem Wirtshaus, häufig auch auf dessen Vorplatz. Für die passende Volksmusik sorgten vorrangig örtliche $\mathrm{Mu}-$ sikgruppen oder eingeladene Musiker aus anderen Gegenden. Diese Veranstaltungen waren zwar den Ledigen vorbehalten, jedoch fanden auch derartige Kulturereignisse für Jung und Alt zu bestimmten Anlässen wie Hochzeiten, Erntefesten und kirchlichen Feiertagen statt. Das Tanzhaus stand somit für einen Ort der Begegnung und zugleich für eine ungezwungene Unterhaltung für Jugendliche auf traditionelle Art und Weise (Martin, 2001, 31-34).

Die siebenbürgische Region durchlebte hinsichtlich ihrer nationalen Zugehörigkeit und ihrer Bevölkerungsgruppen eine äußerst spannungsvolle Geschichte, was sich auch auf die Pflege der Volkskultur auswirkte. Seit dem Vertrag von Trianon 1920 zählte Siebenbürgen zum rumänischen Staatsgebiet, das durch seine heterogene Bevölkerungsstruktur charakterisiert wurde. In dieser Region lebten unterschiedliche ethnische und religiöse Gruppen wie Rumänen, Ungarn, Deutsche, Armenier sowie Christen, Juden, Roma und Sinti. Die größte ethnische Minderheit bildeten die Ungarn, die - trotz oder wegen der Trennung vom Mutterland - ihre ungarischen Bräuche fortführten und weiterhin typische Volkstänze zur entsprechenden musikalischen Begleitung praktizierten. Die Tatsache, dass das ungarische Volkskulturgut in Siebenbürgen bewusst gepflegt wurde, erklärt laut Balázs Balogh und Ágnes Fülemile die Bedeutung dieser Region als wichtigstes Forschungsgebiet für die Anhänger der Tanzhaus-Bewegung in den 1970er Jahren (Balogh und Fülemile, 2008, 50). Die Autoren beteiligten sich selbst in den 1970er und 1980er Jahren an der Rockmusik-Szene sowie der Tanzhaus-Bewegung und untersuchten die sozialen, wirtschaftlichen und kulturellen Prozesse in Siebenbürgen. 


\section{Beispielhafter Ablauf eines Tanzhaus-Abends}

Die erste Tanzhaus-Veranstaltung fand im Jahre 1973 in einem Kulturhaus namens Kassák-Klub in Budapest statt. Die Organisatoren, welche im Übrigen noch heute in der Tanzhaus-Szene aktiv sind, waren Ferenc Sebő und Béla Halmos. Zusammen mit zwei weiteren Musikern bildeten sie das „Sebő-Halmos-Ensemble“. Das Repertoire der Musikgruppe umfasste hauptsächlich Lieder und Tänze aus der Gemeinde Szék, welche den Besuchern durch erfahrene Tanzlehrer, Choreographen und Ethnologen vermittelt wurden. An einem Tanzhaus-Abend konnten die erfahreneren Besucher also nach eigenem Ermessen tanzen, während die Interessierten nebenbei unterrichtet wurden. Laut László Kürti bildeten die regelmäßigen und gut besuchten Tanzhaus-Veranstaltungen in den 1970er Jahren den Ausgangspunkt sowie das Musterbeispiel einer kulturellen Bewegung, welche auch heute noch - allerdings in veränderter Form - aktiv ist (Kürti, 2001, 143).

Durch die Tanzhaus-Bewegung und die gesellschaftlichen Veränderungen wurde das Tanzhaus in verschiedene Kontexte gebracht, der typische Ablauf einer solchen Veranstaltung ist jedoch größtenteils ähnlich geblieben: Eine Gruppe von jungen Menschen im Alter von 15 bis 30 Jahren trifft sich am frühen Abend in einem geeigneten Raum, dem Tanzhaus, um gemeinsam zu traditioneller, akustischer Musik verschiedene Volkstänze zu tanzen. Dies tun sie nicht vorrangig unter dem Aspekt der bewussten und professionellen Volkskulturpflege, sondern weil sie durch den Rückgriff auf Traditionen einen unterhaltsamen und geselligen Zeitvertreib ausüben können. Der Musiker und Musikethnologe Bob Cohen beschreibt den Ablauf eines Tanzhaus-Abends: Im Einführungsteil lehrt ein Tanzpaar traditionelle Volkstänze aus Siebenbürgen oder aus verschiedenen Regionen Ungarns, darunter befinden sich Einzel-, Paar- sowie Kreis- und Reihentänze. Die dazugehörige Musik wird auf traditionellen Instrumenten wie Trommel, Okarina, Türkenpfeife und Bassgeige gespielt. Dieser Unterricht zu Beginn ist notwendig, da nicht vorausgesetzt werden kann, dass alle Besucher die traditionellen Lieder und Tänze bereits kennen. Anschließend wird die Gelegenheit geboten, das eben Gelernte anzuwenden, zu vertiefen und frei zu tanzen, was je nach Laune der Tänzer und Musiker mehrere Stunden lang andauern kann. Danach verweilen die Besucher an der Bar und lassen den Abend mit ungarischem Schnaps und Speckbrot (pálinka und zsíros kenyér) ausklingen (Cohen, 2001).

Kennzeichnend für einen ungarischen Tanzhaus-Abend sind die partizipativen und improvisatorischen Elemente, welche ihn von folkloristischen Darbietungen unterscheiden. Die Besucher beteiligen sich an der Gestaltung der Veranstaltung und bringen eigene Variationen im freien Tanz mit ein. So wird neben dem Praktizieren traditioneller Tänze auch mit den musikalischen Mustern und Tanzschritten experimentiert, wodurch neue, innovative Formen entstehen. 


\title{
1.2 Ungarn zur Entstehungszeit der Tanzhaus-Bewegung
}

\author{
Situation und Bedürfnisse der Gesellschaft
}

In Bezug auf die gesellschaftlichen Verhältnisse zu Beginn der 1970er Jahre ist die Einzigartigkeit des Kádár-Systems im Vergleich zu anderen Ostblock-Staaten hervorzuheben. Eine derartig gelockerte Form des Kommunismus, welche eine gesellschaftliche Liberalisierung zuließ, war beispiellos in Osteuropa. Kádár verfolgte außenpolitisch gesehen eine moskautreue Linie, jedoch gestattete er der ungarischen Bevölkerung vergleichsweise mehr Handlungsfreiraum.

Dadurch wurde ein politischer Kompromiss zwischen der Bevölkerung und der Regierung geschaffen: Zwar musste die politische Situation geduldet werden, dafür wurden jedoch vor allem in wirtschaftlicher und politischer Hinsicht persönliche Freiheiten geboten. Im Rahmen dieser Kompromisshaltung war es der Bevölkerung möglich, sich teilweise selbst zu organisieren. Dies war auch in Bezug auf die Tanzhaus-Bewegung der Fall, da sich ihre Anhänger selbstständig um das Material sowie um die Veranstaltungsräume kümmerten.

Die vergleichsweise tolerante und offene Haltung der Diktatur erlaubte somit die Entstehung jugendlicher Subkulturen, von denen die Beat-Bewegung eine bedeutsame Variante darstellt. Laut Árpád von Klimó fanden erfolgreiche Bands wie Omega oder Illés, die sich an den von der Regierung als „dekadent“ beschimpften Rockgruppen wie den Rolling Stones oder Deep Purple orientierten, großen Zuspruch unter den Jugendlichen. Diese hätten sich somit erstmals seit den Nachkriegsjahren gegen staatliche Verbote und Vorgaben aufgelehnt. Wie Klimó beschreibt, unternahm die kommunistische Regierung entsprechende Bestrebungen, der Beat-Bewegung Einhalt zu gebieten und sie unter Kontrolle zu bringen, um die Propagierung westlicher Werte und Opposition zu verhindern. Diese Kampagnen hätten jedoch mehr Wider- als Abstand erzeugt, sodass sich unter den jungen Ungarn und Intellektuellen eine zunehmende Ablehnung gegenüber der Regierung in verschiedenen halb-offiziellen Subkulturen - etwa der Beat-Bewegung - manifestiert habe. Klimó betont, dass die Beat-Bewegung lediglich eine marginale Gruppe der Bevölkerung bildete, wodurch ihr Einfluss auf die allgemeine Gesellschaft relativ begrenzt blieb (Klimó, 2006, 160-63).

Das zur Entstehungszeit der ungarischen Tanzhaus-Bewegung bestehende Gesellschaftsbild lässt sich folgendermaßen beschreiben: Die nach der ungarischen Revolution von 1956 durch János Kádár gegründete Ungarische Sozialistische Arbeiterpartei (Magyar Szocialista Munkáspárt - MSZMP) konnte ihre drei Hauptziele - Wirtschaftsstabilisierung, Hebung des Lebensstandards sowie die Suche nach Legitimität des Systems - vorerst umsetzen und eine sozialistische Industriegesellschaft etablieren. Im Zuge der Industrialisierung setzte ein Urbanisierungsprozess ein, durch den sich das Arbeiterleben von den ländlichen Agrar- 
landschaften hin zu städtischen Industriegebieten verlagerte. Somit begann auch der Bedeutungsverlust der ländlichen Regionen gegenüber den sich im Wirtschaftsaufschwung befindenden urbanen Gebieten. Mit der zunehmenden Attraktivität der urbanen Gebiete verringerte sich gleichzeitig das Interesse für die ländliche Kultur - das traditionelle Familiensystem mit ihren gemeinschaftlichen Ereignissen wurde aufgelöst und kulturelle Praktiken wie Volksmusik und Volkstanz verloren ihre gesellschaftliche Bedeutung.

\section{Politische Führung und Kulturpolitik}

Tamás Hofer zufolge war die stalinistisch geprägte Kulturpolitik bis 1956 auf die kulturelle Bildung für die breite Masse ausgelegt, was gleichzeitig die unzureichende Unterstützung, teilweise auch beabsichtigte Einschränkung und Unterdrückung des hochqualifizierten Kulturlebens bedeutete. Es galt, eine neue sozialistische Massenkultur zu bilden, welche die nationale Einheit sowie die Annäherung zwischen Arbeiterklasse und Bauerntum symbolisieren sollte. Um dies zu erreichen, habe die Instrumentalisierung der ungarischen Volkskultur mit ihren bäuerlichen Traditionen nahe gelegen. So seien bis 1951 etwa 3000 staatlich unterstützte Tanzgruppen entstanden, welche das von der Kulturpolitik nach sozialistischen Richtlinien zusammengestellte Repertoire aus Volksliedern und -tänzen landesweit aufführten (Hofer, 1992, 272-73). Die Musikwissenschaftlerin Judit Frigyesi merkt an, dass die durch eine entsprechende politische Führung und Kulturpolitik geschaffene ,ungarische Volkskultur“" mit einem Konstrukt vergleichbar sei, welches auf verschiedenen Elementen der Volkskultur beruhe und zeitweise dazu dienen könne, nationalistische, rechtsradikale und faschistische Bestrebungen zu propagieren (Frigyesi, 1996, 57).

Stets sollte ein Gefühl von Optimismus, Fröhlichkeit und Fortschrittlichkeit der Ungarn vermittelt sowie die ungarische Einheit und Größe demonstriert werden. Frigyesi zufolge wurden verschiedene Elemente der Volkskultur, besonders Volkslieder und Tänze, ausgewählt und durch eine entsprechende Inszenierung bühnenreif gemacht. Aus den ursprünglich kleinen Tanzgruppen, welche spontan und improvisierend Volkstänze praktizierten, seien professionelle Ensembles gebildet worden, welche den Eindruck einer auf ungarischer Volkskultur basierenden Veranstaltung erwecken sollten (Frigyesi, 1996, 59). Jedoch repräsentierten die inszenierten und reglementierten Auftritte lediglich das, was als ungarische Volkskultur verstanden und damit vermittelt werden sollte.

Die Ethnographin Linda Dégh, welche die institutionelle Verwendung von Volkskultur in Ungarn untersuchte, vertritt die These, dass Volkskultur politisch instrumentalisiert wurde, um eine kontrollierte öffentliche Meinung zu schaffen und um das staatliche Vorgehen zu legitimieren. Als Beispiel nennt sie das von 
Mátyás Rákosi geführte kommunistische Regime, in dem eine massenwirksame Propaganda betrieben wurde, welche die Ungarn im Sinne des Sozialismus erziehen sollte. Da Volkskultur naturgemäß breiten Bevölkerungsschichten zugänglich sei, habe sie gezielt als Mittel der Massenerziehung eingesetzt werden können, um die Moralvorstellungen sowie die sozialen und beruflichen Beziehungen der Bevölkerung zu beeinflussen. Die zwischen 1948 und 1956 in Ungarn präsentierte Volkskultur habe demnach nicht auf Forschung und Überlieferung beruht, sondern bildete das Ergebnis der von der Regierung in Auftrag gegebenen Arbeiten professioneller Musiker. Ethnologen wurden laut Dégh als Propagandisten eingesetzt, mit deren Hilfe eine nationale Volkskultur geschaffen werden sollte. Eine ungarische Volkskultur, welche jedoch staatlich kontrolliert und stilisiert wurde, wie zum Beispiel in den Bühnenpräsentationen des „Ungarischen Staatlichen Fokloreensembles“ zum Ausdruck kam (Dégh, 1984, 202-203).

Die ungarische Volkskultur als repräsentative Nationalkultur war demnach seitens des Staates mit einer sozialistischen Ideologie besetzt, welche durch die diversen staatlich geförderten Institutionen wie dem 1951 gegründeten „Ungarischen Staatlichen Folkloreensemble“ umgesetzt wurde. Laut Hofer entstand aufgrund der demonstrativ hohen Bewertung volkskultureller Elemente im Sinne des „Sozialistischen Folklorismus“ eine ideologische Überfrachtung und Engstirnigkeit hinsichtlich real existierender kultureller Entwicklungen. Diese sei unter der Kulturpolitik János Kádárs gelockert worden. Ab 1956 seien politische Veranstaltungen nicht mehr zwangsläufig von Folklorevorführungen im sozialistischen Volkskunststil begleitet, sondern mehr Raum zur freien kulturellen Betätigung geboten worden, soweit keine oppositionelle Haltung zum Ausdruck gebracht wurde (Hofer, 1992, 272-73).

Das allgemeine Bild von Volkskultur wurde nicht nur durch die Kulturpolitik, sondern auch durch das ungarische Bildungswesen geprägt. Judit Frigyesi verweist auf die Probleme der ideologisch motivierten Verwendung ungarischer Volkskultur - oder dem, was durch die Kulturpolitik als solche deklariert wurde. Aufgrund der Tatsache, dass Volkskultur im Sinne des Sozialismus für die breite Masse zugänglich gemacht werden sollte, sei ihr im ungarischen Bildungssystem entsprechender Raum geboten worden: Volksmusik wurde - in vereinfachter Form - als Pflichtfach an den Schulen eingeführt. Frigyesi kritisiert, dass den Schülern und Studenten eine umfassende Kenntnis über Volksmusik und Lieder abverlangt wurde, während sich ihnen kaum eine Gelegenheit bot, einen Eindruck von Aufführungen in ihrem ursprünglichen Kontext zu gewinnen und sie selbst zu erleben. So sei ein umfangreiches und doch staatlich vorselektiertes Wissen über die ungarische Volksmusik vermittelt worden, jedoch habe diese theoretische und exzessive Lehre dazu geführt, dass sie den jungen Menschen als leblos-langweiliges Material erschien. Aufgrund des fehlenden Kontextes sei demnach die Vorstellung von Volksmusik als eine Unterhaltungsform, die der Praxis und nicht der 
Theorie dient, gar nicht erst entstanden. Weiterhin beschreibt Frigyesi die innerhalb der Bevölkerung aufkommende Abstandshaltung zur Volksmusik, welche in der allgemeinen Ansicht begründet lag, dass die inszenierte Volkskultur als Werkzeug für ideologische Steuerung zu meiden sei und für das moderne Leben keine Rolle mehr spiele. Diese Situation habe die Grundlage für die Aktivitäten verschiedener Bewegungen zur Wiederbelebung ungarischer Traditionen, so auch der Tanzhaus-Bewegung gebildet. Deren Anhänger wendeten sich gezielt der praktischen Seite von Volksmusik zu und legten besonderen Wert darauf, sie von den bäuerlichen Musikern direkt oder von Musikern, die mit ihnen in Kontakt standen, zu lernen. Volksmusik und Volkstanz sollten wieder ihre eigentliche Funktion erfüllen: für eine ungezwungene Unterhaltung sorgen (Frigyesi, 1996, 64-67).

\subsection{Vom Volksfest zur Tanzhaus-Bewegung}

\section{Auf der Suche nach dem Ursprünglichen}

Im Gegensatz zur ungarischen Beat-Welle der 1960er Jahre, die sich an westlichen Werten orientierte und sich einer neuen musikalischen Sprache zum Ausdruck der Befindlichkeit sowie der Auflehnung gegen gesellschaftliche Konventionen bediente, wendete sich die Tanzhaus-Bewegung hin zu den eigenen ungarischen Werten. Nach der Zeit, in der die „ungarische Volkskultur“ durch den Staat gefördert, aber auch gleichzeitig gelenkt wurde, begann eine Periode, in der Intellektuelle begannen, sich für das zu interessieren, was gerade noch nicht in staatlichen Händen lag. Es bestand ein besonderer Bedarf an Spontaneität und Improvisation, letztendlich an Freiheit, welche durch die vorgeschriebenen Kulturprogramme und staatlichen Reglementierungen nicht möglich waren.

Ab den 1960er Jahren gab es laut ungarischen Soziologen eine Reihe an verschiedenen spontanen kulturellen Aktivitäten, die von Jugendlichen initiiert wurden und sich schnell verbreiteten. Frigyesi fasst sie wie folgt zusammen: Die erste Welle repräsentierte die Beat-Bewegung, welche sich an westlicher Rock-Musik orientierte. Die zweite Welle wurde durch die experimentelle Auseinandersetzung mit dem ungarischen Theater der Avantgarde gebildet. Sie entstand zu Beginn des 20. Jahrhunderts, ihre Hochzeit bestand zwischen 1910 und 1930. Einer der wichtigsten Avantgarde-Autoren war Lajos Kassák, nach dem auch der Budapester Klub benannt ist, der zum Schauplatz der ersten Tanzhaus-Veranstaltungen wurde. Die dritte Periode wurde durch die Tanzhaus-Bewegung charakterisiert, welche eine Alternative zur Beat-Musik darstellte und aus dem Experimentieren mit avantgardistischen Gedichten hervorging (Frigyesi, 1996, 60-61).

Die Suche nach dem Ursprünglichen entstand aus Unzufriedenheit mit der staatlichen Präsentation von Volkskultur. Vor allem junge Intellektuelle gaben 
sich Anfang der 1970er Jahre nicht mehr damit zufrieden, permanent eine Version von Volkskultur dargeboten zu bekommen, die hauptsächlich darauf ausgerichtet war, die ungarische Bevölkerung als stolze und fröhliche Einheit darzustellen. Mit dem Glauben daran, dass Volkskultur mehr als diese reduzierte, bereinigte Variante zu bieten vermag, begaben sich Studenten und Wissenschaftler auf die Suche nach dem, was bisher nicht mit in das ungarische Kultur- und Bildungswesen einbezogen wurde. In der Volksmusik und in den Volkstänzen, welche sie in den siebenbürgischen Dörfern vorfanden, war schließlich eine Form von Volkskultur zu entdecken, wie sie aufgrund der ideologischen Verformung bisher nicht bekannt war. Dabei ist darauf zu verweisen, dass sich unter den Wissenschaftlern auch Komponisten und Choreographen befanden, welche im Auftrag des Staates neues Material aus den Dörfern sammeln sollten, um es mit in die Programme einflechten zu können.

Eine zentrale Rolle hinsichtlich der neuen Tendenzen des Volkstanzes nahm Sándor Tímár ein, der durch seine Aktivitäten in der Tanzhaus-Bewegung sowie durch das Unterrichten von Volkstänzen weltweite Anerkennung erlangte. Er war von 1958 bis 1981 Choreograph des „Bartók-Ensembles“ sowie des „Ungarischen Staatlichen Folkloreensembles" von 1981 bis 1996. In dieser Position konnte er maßgeblich zur Verbreitung der Tanzhaus-Bewegung beitragen.

Frigyesi beschreibt, wie Tímár zu Beginn der 1970er Jahre versuchte, die reglementierten Formen der Bühnenvorführungen von Volkstänzen aufzubrechen. Er habe die Intention verfolgt, einen neuen Stil einzuführen, dessen Schwerpunkt auf Improvisation und Spontaneität, also auf künstlerischer Freiheit liegen sollte. Die dazu passende Musik sei dementsprechend nicht in Form von akademischen Kompositionen zu finden gewesen, sondern musste durch Feldforschung in den verschiedenen ungarischen Regionen inner- und außerhalb der staatlichen Grenzen erforscht werden. Musikalische Unterstützung habe Tímár bei dem „Sebő-Halmos-Ensemble“ gefunden. Béla Halmos und Ferenc Sebő hätten zwar nicht zu den professionellsten Musikern gehört, doch gerade dieser Umstand habe es ihnen möglich gemacht, sich auf Improvisationen nach den Mustern der traditionellen Volksmusik einzulassen (Frigyesi, 1996, 59-60). Das Element der Improvisation charakterisierte das ungarische Tanzhaus und unterschied es somit von den üblichen Musik-Veranstaltungen.

Cohen zeigt einen weiteren Faktor für die Suche nach dem Ursprünglichen auf und betont in seiner Beschreibung des „Sebő-Halmos-Ensembles“ die besondere Wertlegung auf Traditionen. Im Angesicht des Modernisierungsprozesses scheinen diese langsam zurückgedrängt zu werden, was eine neue Aufmerksamkeit erzeuge. Nicht nur die Umgestaltung des kommunistischen Gesellschaftsbildes, in dem die Bräuche und Traditionen der einfachen Bauern infolge der Kollektivierung und Technologisierung keinen Platz mehr hatten, habe zum Bedeutungsverlust der bisher für die ungarische Nation wesentlichen Volkskultur geführt. Be- 
sonderen Einfluss habe auch die Veränderung der ungarischen Medienwelt ausgeübt, die durch Fernsehen und Radiosendungen die selbst geschaffene Unterhaltung mittels Volksmusik in den Hintergrund rückte (Cohen, 2001).

Diese Entwicklungen lassen die Behauptung zu, dass bei einem Teil der jungen Bevölkerung eine gegenteilige Bewegung ausgelöst wurde, deren Ziel es war, die im Verschwinden begriffenen ungarischen Traditionen am Leben zu erhalten oder wiederzubeleben - und durch innovative Elemente zu „erneuern“. Durch Musikgruppen wie das „Sebő-Halmos-Ensemble“ wurden interessierte Jugendliche dazu animiert, sich mit traditioneller Musik und Volkstänzen der ungarischen Dörfer zu beschäftigen. Es kam zu intensiven Begegnungen zwischen jungen Stadt- und älteren Dorfbewohnern, bei denen die Elemente der ungarischen Volkskultur überliefert wurden, welche noch nicht durch Industrialisierung oder professionelle Instrumentalisierung verändert worden sind.

Im Unterschied zum Folklorismus, bei dem das Reservoir der Volkskultur vor allem für wirtschaftliche und kulturindustrielle Zwecke genutzt wird, hatte die Suche nach dem Ursprünglichen andere Hintergründe: zum einen die Übersättigung an Volkskultur durch die „sozialistische Folklore“ und zum anderen die durch Modernisierung hervorgerufene Schnelllebigkeit der Gesellschaft. Traditionelle Musik und Tänze sind nicht nur erlernbar, sondern auch erlebbar, was einen erheblichen Unterschied zu den Bühnenproduktionen der staatlichen Ensembles darstellt, die zwar zum Vorführen, aber nicht zum Teilnehmen dienten. Zudem spielt sich die Reproduktion von Volkskultur in einem gesellschaftlichen Kontext ab, der ein Gemeinschaftsgefühl voraussetzt - eine Prämisse, die auf Freiwilligkeit beruhen sollte, es jedoch im reglementierten Gesellschaftssystem Ungarns zu Zeiten des Kommunismus nicht tat. Wie das auf der Suche nach dem Ursprünglichen gefundene traditionelle Kulturgut vom Dorfe in die Stadt getragen wurde, wird im Folgenden beschrieben.

\section{Das Tanzhaus zieht vom Dorfe in die Stadt}

In einem Artikel beschreibt László Kelemen, der sich seit den 1970er Jahren an der Tanzhaus-Bewegung beteiligt und das ungarische Kulturprojekt Hungarian Heritage House (Hagyományok Háza) leitet, wie der Grundstein für die Tanzhaus-Bewegung gelegt wurde. Dabei wird die Rolle seiner Kollegen Ferenc Sebő und Béla Halmos sowie der Choreographen György Martin und Sándor Tímár besonders hervorgehoben. Sie studierten die ungarische Volksmusik, indem sie Kontakt mit den Musikern der ungarischen Dörfer Siebenbürgens aufnahmen. Durch Zuschauen, Zuhören und Nachahmen der Musik, die sie auf den Tanzhaus-Veranstaltungen sowie Festlichkeiten erlebten, konnten sie das Erlernte in ihren selbst organisierten städtischen Tanzhaus-Veranstaltungen weitergeben. 
Dieser neuartige Zugang zu ungarischen Traditionen erzielte großen Erfolg unter jungen Menschen, denn erstmalig durften sie die Tänze nicht nur auf der Bühne bewundern, sondern sie selbst erlernen und praktizieren. Diese neue Erfahrung stieß laut Kelemen bei vielen jungen Menschen auf reges Interesse, sodass sie selbst in die abgelegenen Dörfer zogen und die dort praktizierte, gelebte Volkskultur „sammelten“. So lernten sie sowohl die Tänze als auch die Spielweise auf den traditionellen Instrumenten kennen. In den folgenden Jahren seien mehrere Musikgruppen entstanden, welche die ungarische Volksmusik so vermittelten, wie die Musiker sie in den Dörfern selbst erlebt haben. Weiterhin seien große Treffen und Arbeitskreise organisiert worden, bei denen das volkskulturelle Wissen in großem Maße von den Praktizierenden an die Interessierten weitergegeben werden konnte (Kelemen, 2004).

Die Musiker der Tanzhaus-Bewegung waren sich dessen bewusst, dass sie den ursprünglichen dörflichen Kontext in einer Stadt nicht würden herstellen können, jedoch erhielten Volksmusik und -tanz nach den vielen Jahren, in denen sie praktisch nur auf der Bühne zu sehen waren und als nur den Künstlern vorbehalten galten, ihre ursprüngliche Funktion zurück: Unterhaltung durch gemeinschaftliches Tanzen. Demzufolge wurde Traditionelles wieder von jungen Menschen angenommen und aufgegriffen.

Frigyesi hebt hervor, dass die Betonung eindeutig auf den Darbietungen, auf Improvisation und Tanz - nicht auf musikalischer Perfektion lag. Es sei nicht das Anliegen der Musiker gewesen, Volksmusik zu praktizieren, um sie somit vor dem Verschwinden zu bewahren. Vielmehr sei es das neuere Interesse für Volkskultur gewesen, welches sie zur Beschäftigung mit ungarischen Traditionen veranlasste. Da es Stadtbewohner waren, die diesem Interesse nachgingen, konnten dörfliche Traditionen in ein neues, städtisches Umfeld gebracht werden, wo sie weitergeführt wurden und damit erhalten blieben (Frigyesi, 1996, 67).

Die jungen Musiker, welche die traditionellen Techniken und Stile dort gelernt haben, wo Volksmusik und Volkstanz entstanden sind, bildeten laut Balogh und Fülemile das erste Glied einer neuen Überlieferungskette, die ihren Weg in den Städten in Form der Tanzhaus-Bewegung fortsetzte. Somit sei das reiche Repertoire sowie die hohe Qualität der Musik und der Tänze in den folgenden Tanzhaus-Generationen übernommen worden. Wie das „Sebő-Halmos-Ensemble“, „Muzsikás“ und das „Bartók Volkstanz-Ensemble“ hätten viele weitere Profiund Amateurbands die Volksmusik neu entdeckt. In den 1970er Jahren seien in Budapest und anderen Großstädten zahlreiche Klubs entstanden, in denen zur Musik der Bands getanzt wurde und deren Türen allen offen standen - sowohl Studenten als auch Arbeitern. Gemeinsam hätten sie über ihre eigenen Traditionen gelernt, mit der sie nicht mehr innerhalb ihrer Familien - wie in den Dörfern, wo das traditionelle Familienmodell noch existierte -, sondern innerhalb der Gruppe in Berührung kommen konnten. Balogh und Fülemile schlussfolgern da- 
raus, dass durch die Tanzhaus-Bewegung nicht nur musikalisches Wissen und Können vermittelt, sondern auch ein neues Bewusstsein geschaffen wurde. Somit wurden Musik und Tanz als Bestandteile einer komplexen und integrativen Kultur an sich neu entdeckt und geschätzt (Balogh und Fülemile, 2008, 47).

Was Sebő und Halmos im Hinblick auf die Volksmusik unternahmen, wurde in ähnlicher Weise von Sándor Tímár und György Martin in Bezug auf den Volks$\operatorname{tanz}$ betrieben. Sie haben die verschiedenen Tänze studiert und die unterschiedlichen Aussagen und Bedeutungen der Tanzschritte - die Sprache der Tänzer - zu erkennen gelernt. Der Choreograph und Volkstanz-Lehrer László Diószegi betont die Tatsache, dass den Tänzen keine Choreographie zugrunde lag, sondern improvisiert wurde. Dies forderte sie bei ihren Studien umso mehr heraus, da die Tänze nicht einfach auswendig gelernt werden konnten. Es sei wichtig gewesen, die Geschichten und Situationen zu verstehen, die Emotionen zu fühlen, um die entsprechende Ausdruckskraft in die Tanzschritte legen zu können. Tímár und Martin hätten eine effektive Methode entwickelt, mit der sie ihren Schülern die Volkstänze und die Kunst des improvisierenden Tanzens näher bringen konnten. Dabei hätten sie in den 1970ern einen so großen Erfolg erzielt, dass sie im In- und Ausland eingeladen wurden, um ungarischen Volkstanz zu unterrichten. Das von Tímár geleitete „Bartók-Ensemble“ habe jedes Jahr neue Choreographien herausgebracht, welche von unzähligen Tanzstilen und regional unterschiedlichen Volkstanztraditionen inspiriert wurden. Besonders das Karpatenbecken habe für viele als eine reiche Quelle an neuen Entdeckungen gegolten. Dementsprechend wurde Volkskultur nicht mehr durch die parteilich geleiteten Institutionen in den Städten konstruiert, sondern in den Dörfern entdeckt und erforscht, um sie anschließend als traditionelles Kulturgut in die Städte zu tragen und dort bekannter zu machen (Diószegi, 2008, 7-8).

Mit der geographischen Verlagerung des traditionellen Volkstanzes in einen urbanen Raum, in dem sich die Tanzhaus-Bewegung entwickeln konnte, ging auch eine Veränderung der Bedeutung von ungarischem Volkstanz in seinem neuen Kontext einher. Auf ländlichem Gebiet praktiziertes traditionelles Tanzen und Feiern galt als eine Selbstverständlichkeit, wohingegen es in der Großstadt, wo jegliche Konzentration auf Fortschritt und Modernisierung lag, eher einer Provokation ähnelte. In dieser Hinsicht kommt der Aspekt der unterschiedlichen Auffassungen von Volkskultur zum Tragen.

\section{Von der Unterhaltung zur Opposition}

Hofer prägte in Bezug auf die politische Nutzbarmachung von Elementen ungarischer Volkskultur den Begriff der „sozialistischen Folklore“. Sie habe dem Zweck des Zusammenhalts und des gemeinschaftlichen Aufbaus der sozialisti- 
schen Gesellschaft in Ungarn gedient. In diesem Falle habe die Regierung mit volkskulturellen Mitteln, zum Beispiel Volksliedern und Bühnentänzen, gearbeitet, welche die ungarische Gesellschaft als fleißiges und fröhliches Volk demonstrieren sollten. Das Reservoir der Volkskultur, vor allem Musik und Tanz, sei jedoch auch von der oppositionellen Seite verwendet worden, um ihre neuen Sichtund Denkweisen zu verbreiten, denn es habe genügend Spielraum für subtile Botschaften gelassen, die von den jeweiligen Akteuren unterschiedlich ausgelegt werden konnten. Durch Musik und Tanz als Medium war es dadurch möglich, politische, ethische und soziale Einstellungen darzustellen, deren öffentliche Artikulation politische Folgen nach sich gezogen hätte (Hofer, 1992, 273).

Laut Frigyesi herrschten in der ungarischen Öffentlichkeit der 1970er Jahre diverse, teilweise gegensätzliche Meinungen und Einstellungen zur aufkommenden Tanzhaus-Bewegung: Einige sahen in ihr das Wiederaufleben ungarischer Popmusik, die den zunehmenden Einfluss westlicher Musikstile wie den Rock 'n Roll verdrängen sollte - ein Eindruck, der bereits aufgrund des experimentellen Umgangs mit neuen musikalischen Elementen widerlegt wurde. Andere interpretierten die neue Form des improvisierenden Musizierens als Konkurrenz für die klassische Methode, welche an den Musikhochschulen gelehrt wurde. Weitere schrieben den Anhängern der Bewegung nationalistische Tendenzen zu, die sie mit der intensiven Beschäftigung mit ungarischer Musik aus Siebenbürgen als Ausdruck revisionistischer Einstellungen begründeten (Frigyesi, 1996, 55-56). Die Teilnehmer selbst hätten die Tanzhaus-Bewegung jedoch als eine neue Form von Unterhaltungsmusik mit partizipativem Charakter und einer sekundären Funktion hinsichtlich der subtilen Kommunikation nationalistischer Ansichten und Forderungen beschrieben. Frigyesi bezweifelt, dass die Tanzhaus-Bewegung seitens der Regierung jemals öffentlich unterdrückt worden sei, allerdings räumt sie ein, dass sie unter dem Verdacht der Opposition standen und deswegen ungern von Kulturzentren unterstützt wurden (Frigyesi, 1996, 71).

Offensichtlich bestand ein bemerkenswerter Unterschied zwischen der Einschätzung der Anhänger der Bewegung und der öffentlichen Interpretation, weshalb eine Untersuchung der Aussagen der Gründer der Tanzhaus-Bewegung angebracht ist. Tamás Szőnyei, welcher sich mit der ungarischen Rockmusik-Szene und verschiedenen Subkulturen beschäftigte, verweist auf Ferenc Sebő, welcher immer wieder betonte, dass die Tanzhaus-Veranstaltungen keineswegs nationalistische Bestrebungen zum Ausdruck bringen sollten. Vielmehr sei das musikalische Material nach künstlerischen Aspekten ausgewählt worden, um einen unterhaltsamen Abend mit schönen Liedern für junge Menschen zu gestalten. Des Weiteren hätte die Konzentration der Musiker nicht auf der vollständigen Erhaltung der Traditionen gelegen, was sich auch daran zeigte, dass die Tanzhaus-Veranstaltungen nicht nur zu bestimmten Anlässen stattfanden und die Teilnehmer nicht in entsprechenden Trachten auftraten, sondern in bequemer Alltagsklei- 
dung. Es wurde getanzt, wenn man in der Laune dazu war - ohne sich am Kirchenkalender orientieren und in traditionelle Kleidung zwängen zu müssen (Szőnyei, 2005, 86-89).

Den Vorwurf der nationalistischen Tendenzen konnte Béla Halmos ebenfalls zurückweisen, schließlich wurden die traditionellen Kulturgüter der nicht-magyarischen Völker Ungarns gleichwertig betrachtet. So wurden auch rumänische, serbische, kroatische und weitere Musik und Tänze in das Repertoire der Tanzhaus-Gruppen aufgenommen. Die Hauptsache bestand laut Balogh und Fülemile darin, dass die ethnischen Traditionen der auf ungarischem Gebiet lebenden Volksgruppen sowie der im Ausland lebenden Minderheiten ,authentisch“ und „ursprünglich“ wirkten. Die Tanzhaus-Bewegung setzte sich demnach bewusst mit verschiedenen Ethnien auseinander und war folglich durchaus offen für nicht-magyarische Einflüsse (Balogh und Fülemile, 2008, 49-50).

Die ersten und entscheidenden Träger der Tanzhaus-Bewegung legten erheblichen Wert auf den Unterhaltungsaspekt der Veranstaltungen, für die sie ihre Inspirationen in der Musik und in den Tänzen der ungarischen Volkskultur fanden. Nicht das Produkt Volkskultur, sondern der Prozess stand im Mittelpunkt des Tanzhaus-Abends, bei dem die eigens ausgewählten Traditionen auf eine neue Art und Weise ausgelebt wurden. Dabei stellt sich die Frage, worin das „Oppositionelle“ der Tanzhaus-Bewegung lag und weshalb sie als „halb-legale Subkultur" bezeichnet wurde.

In Bezug auf den Verdacht der Nonkonformität mit der politischen Linie Ungarns sind mehrere wichtige Anhaltspunkte zu nennen: Erstens lautete das Motto der Zeit und damit auch der Tanzhaus-Bewegung: ,Werdet selbst aktiv und lasst euch nicht von den Älteren vorschreiben, was ihr tun sollt!“”(Ronström, 1998, 3). Die jungen Menschen handelten selbstständig und organisierten sich selbst, was die staatlichen Möglichkeiten zur Einflussnahme verringerte. Diese Unkontrollierbarkeit führte dazu, dass die Bewegung von der Regierung als „gefährlich“ eingestuft wurde. Die Beschäftigung mit Volkskultur stellte folglich eine Alternative zum staatlich organisierten, kommunistischen Freizeitangebot dar. Für einen Tanzhaus-Abend bedurfte es weder staatlicher Unterstützung noch formalisierter Bühnenshows, denn sowohl die Musik als auch die Tänze wurden von den Teilnehmenden selbst bestimmt, produziert und praktiziert.

Zweitens verkörperte die Tanzhaus-Bewegung ein Lebensgefühl, welches auf dem Alten und Traditionellen beruhte und gleichzeitig sehr lebendig und frisch wirkte. Dies erschien für Unbeteiligte als Kontrast zum allgemeinen Streben nach Neuem und Fortschrittlichem. Allerdings waren die Mitglieder der Tanzhaus-Bewegung ebenfalls an Neuem interessiert, wie der Musiker und Ethnologe Owe Ronström äußert. Die selbst entdeckte und bisher unbekannte Volkskultur stellte durchaus etwas Neuartiges dar. Dementsprechend hätten die jungen Ungarn keineswegs eine Flucht in die Vergangenheit oder Rückwärtsgerichtetheit beabsich- 
tigt. Vielmehr hätten sie das bereits Bestehende in die Gegenwart mit eingebracht (Ronström, 1998, 3-4).

Drittens wurde die staatliche Version von ungarischer Volkskultur nicht mehr akzeptiert, wie Balogh und Fülemile behaupten. Deshalb hätten sich die Mitglieder der Tanzhaus-Bewegung eigenständig auf Forschungsausflüge begeben und nach kontextbezogener Volkskultur gesucht, welche sie zunächst in den abgelegenen Dörfern in Siebenbürgen zu finden hofften. Das eigene Handeln drückte die kritische und ablehnende Haltung der Jugendlichen gegenüber der ,,sozialistischen Folklore" aus, welche sie durch die verpflichtende Mitgliedschaft in Jugendorganisationen, bei Schulfeiern und zu politischen Demonstrationen vorgeführt bekamen. Folglich kann die Tanzhaus-Bewegung als Gegenkultur bezeichnet werden, denn sie bildete einen Gegenpol zur kommunistischen Kulturpolitik. Wie Balogh und Fülemile hinzufügen, habe besonders die Solidarität mit der in Rumänien lebenden, unterdrückten ungarischen Minderheit, welche durch das Praktizieren der Siebenbürgischen Tänze und Musik demonstriert wurde, den kommunistischen Regierungen in Ungarn und Rumänien Grund zum Missmut gegenüber der Tanzhaus-Bewegung gegeben (Balogh und Fülemile, 2008, 48-50).

Ausgehend von den bisher genannten Argumenten lässt sich folgende Einschätzung formulieren: Der Begriff der Tanzhaus-Bewegung taucht immer wieder in Verbindung mit politischer Aktion auf, sodass ihr keine politische Neutralität zugeschrieben werden kann. Zwar betonten die einzelnen Begründer der Bewegung stets den Aspekt der Unterhaltung und der damit einhergehenden Wiederbelebung ungarischer Volkskultur. Jedoch kann eine kritische Einstellung gegenüber der Regierung nicht zurückgewiesen werden, da die Aktivitäten der Tanzhaus-Anhänger - die Selbstorganisation und die Vermittlung von einem anderen Bild von Volkskultur - an sich bereits nicht parteikonform waren. Die Entwicklung der Tanzhaus-Bewegung konnte aufgrund ihrer Unabhängigkeit und Selbstständigkeit nicht unter staatliche Kontrolle gebracht werden, sodass sie als „gefährlich“ deklariert wurde. Dies ist jedoch ein Etikett, welches der Tanzhaus-Bewegung von außen zugeschrieben wurde. Die Tanzhaus-Enthusiasten, welche sich in den 1970er und 1980er Jahren aktiv an der Bewegung beteiligten, taten dies demzufolge in dem Bewusstsein, dass ihr Handeln als regierungskritisch eingestuft werden konnte, selbst wenn ihnen der kulturelle Aspekt wichtiger war als der politische. Darüber hinaus ist zu betonen, dass die Tanzhaus-Bewegung im Vergleich zur Beat- und Rock-Szene bedeutend weniger Anhänger vereinte und somit keine echte Gefahr für das politische System darstellen konnte. 


\title{
2. Das ungarische Tanzhaus - Volkskultur in der Moderne
}

\subsection{Zur Umdeutung des Entdeckten und Erlernten}

\author{
Traditionelles in neuer Form
}

Inwiefern die Tanzhaus-Bewegung eine neuartige Form der traditionellen Volkskulturpflege darstellt, soll anhand einer Gegenüberstellung vom ursprünglichen, dörflichen Tanzhaus und der neueren, städtischen Form gezeigt werden. Wie bereits beschrieben, transportierte die Tanzhaus-Bewegung Volksmusik und -tänze von den ungarischen Dörfern in die Großstädte, sodass die Veranstaltungen nicht mehr in einem Wirtshaus oder gemieteten Bauernhaus stattfanden, sondern in diversen Klubs und Veranstaltungshäusern. Während auf dem Lande zur Unterhaltung für junge Männer und Frauen oder zu verschiedenen kirchlichen Feier- und Festtagen getanzt wurde, gab es in den Städten keinen bestimmten Anlass für einen Tanzhaus-Abend außer die Freude am Tanzen sowie das Interesse für die traditionellen Lieder und Tänze. Die Dorfbewohner lernten die regional typischen Tänze und Lieder von Kindesalter an, wohingegen die Anhänger der Tanzhaus-Bewegung den direkten Kontakt zu den Musikern und Tänzern suchen mussten, um deren Kenntnisse und Fähigkeiten übernehmen zu können. Weiterhin bot eine dörfliche Tanzhaus-Veranstaltung eine der wenigen Gelegenheiten zur gemeinschaftlichen Unterhaltung, wobei der Aspekt der bewussten Traditionspflege stets bedeutsam war. Als das Tanzhaus in die Städte kam, spielte zwar auch die Verbreitung, besonders das Wiederbeleben der Traditionen eine große Rolle, jedoch wurde es vom Interesse am Experimentieren mit der neu entdeckten ungarischen Volkskultur geleitet. Schließlich sind zwei formale Unterschiede zu nennen: Zum einen beruht die Darbietungsform der Musik und Tänze nicht mehr auf auswendig gelernten Überlieferungen, die von Generation zu Generation weitergegeben wurden, sondern auf Grundlagen, welche die Anhänger der Tanzhaus-Bewegung von den Dorfbewohnern übernommen und anschließend durch improvisatorische Elemente ergänzt haben. Zum anderen wurde an den traditionellen Feier- und Festtagen, an denen Tanzhaus-Veranstaltungen stattfanden, die entsprechende Kleidung getragen, nämlich die für die jeweilige Region typische Tracht. Da die urbane Version des Tanzhauses nicht an offizielle Festlichkeiten gebunden war, mussten auch keine passenden Kleider getragen werden, sodass die Besucher einer Tanzhaus-Veranstaltung meistens in bequemer Alltagskleidung erschienen.

Im Hinblick auf die Umdeutung des Entdeckten und Erlernten ist festzuhalten, dass die Tanzhaus-Bewegung sowohl Traditionsbehaftetes als auch Neuartiges vereint. Indem Tanzhaus-Veranstaltungen weiterhin ihre Funktion als Begeg- 
nungsort und Unterhaltungsform beibehalten und durch die traditionellen Elemente der ungarischen Volkskultur, vor allem Tänze, Musik und Instrumente, gestaltet werden, bleibt der Aspekt der Traditionspflege erhalten. Jedoch finden Tanzhaus-Abende in einem neuen Kontext statt, welcher durch Urbanität und Modernität geprägt ist. In einer Großstadt haben Jugendliche eine umfangreiche Auswahl zwischen verschiedenen Unterhaltungsformen, welche in den Dörfern nicht gewährleistet werden konnte. Im urbanen Raum wird demzufolge ganz bewusst auf Traditionen zurückgegriffen, um eine Alternative zum bestehenden Kulturangebot zu schaffen und um die vom Fortschrittsdenken verdrängten Güter der ungarischen Volkskultur in neuer Form, nämlich als Retrokultur, wiederzubeleben: Die Tanzhaus-Bewegung steht demzufolge für Traditionelles in T-Shirt und Turnschuhen - die an moderne Ansprüche angepasste Form der Volkskulturpflege.

\section{Aus der Gegenkultur wird ein Kulturevent}

Dégh betont, dass sich ein wesentlicher Kritikpunkt der Tanzhaus-Enthusiasten auf die offizielle, durch die ungarische Kulturpolitik geschaffene Auffassung von Volkskultur als Ausdruck einer kollektiven kulturellen Identität bezog: Im Stalinismus sollten die Bevölkerungsschichten unter dem Decknamen von Volkskultur und dem aus ihr hervorgehenden gemeinsamen kulturellen Erbe vereint werden, um somit eine gemeinschaftliche sozialistische Ausrichtung von Kultur zu gewährleisten. Die Anhänger der Tanzhaus-Bewegung setzten sich demgegenüber für eine Wahrnehmung von Volkskultur als vielseitige, durch regionale und soziale Gegebenheiten charakterisierte Kulturform ein. Erst im Rahmen der wesentlich liberaleren Kulturpolitik unter Kádár ergaben sich laut Dégh mehr Möglichkeiten, im kulturellen Bereich tätig zu werden und einen individuellen, innovativen Zugang zu Volkskultur zu bieten sowie neue Trends zu setzen (Dégh, 1984, 208-209).

Wie aus den Ausführungen von János Balázs bezüglich der ungarischen Volksmusik-Bewegung als Jugendsubkultur hervorgeht, entwickelte sich die ursprünglich als Gegenkultur entstandene Tanzhaus-Bewegung nach einigen Jahren zu einer offiziell etablierten Freizeitbeschäftigung, welche staatliche Unterstützung erhielt. Die Tanzhaus-Veranstaltungen wurden durch ein umfangreicheres Rahmenprogramm ergänzt. Beispielsweise fanden zusätzlich Lesungen und Diskussionen zu Völkerkunde, Literatur und Kunst statt, wobei der Tanz-Teil stets die bedeutendste Rolle spielte. Wie Balázs einschätzt, avancierten die Veranstaltungen zu einer sehr beliebten Freizeitaktivität junger Ungarinnen und Ungarn. In den 1970er Jahren seien zahlreiche Tanzhaus-Klubs entstanden und derartige Veranstaltungen in das Programm der Kulturhäuser aufgenommen worden - sowohl in den Städten als auch in den Dörfern. Balázs behauptet, dass die zu- 
nehmende Popularität der Tanzhaus-Bewegung von den führenden Kulturpolitikern wahrgenommen und als Zeichen für das Wiederaufleben einer Volks- und Nationalkultur überschätzt wurde. An dieser Stelle habe sich der Staat unterstützend eingebracht, indem mithilfe der Massenmedien und den Einrichtungen für Erwachsenenbildung gezielt über die Bewegung und deren Anliegen zur Wiederbelebung der ungarischen Volkskultur informiert worden sei. Durch das staatliche Eingreifen wurde die Tanzhaus-Bewegung demnach als offizielle Kultur deklariert, was gleichzeitig ihren oppositionellen und subkulturellen Charakter verdrängte. Die Tanzhaus-Amateure hatten dadurch die Chance, zu professionellen Musikern zu avancieren, wie es das Beispiel von „Muzsikás“ bereits gezeigt hat. Jedoch ist anzunehmen, dass die Unterzeichnung eines Arbeitsvertrages eine Absage an die unabhängige Tätigkeit als Tanzhaus-Musiker zugunsten des Erfolges in der offiziellen Kulturszene bedeutete. Wie Balázs anmerkt, herrschte in den 1980er Jahren ein regelrechter Wettkampf um Anerkennung. Die Musiker und Gruppen, welche weiterhin auf ihre Unabhängigkeit und eigene Kreativität bestanden und damit beweisen wollten, dass hochgradige Musik auch auf der Grundlage von Volksmusik geschaffen werden kann, riskierten somit ihre musikalische Karriere (Balázs, 1983, 40-41).

Ein weiterer Aspekt, der den Wandel der Tanzhaus-Bewegung von einer Gegenkultur zum Kulturevent unterstreicht, bezieht sich auf den Generationswechsel. Ronström erklärt, dass ein deutlicher Unterschied zwischen den Anliegen der Gründungsgeneration und den nachfolgenden Generationen besteht. Während die Tanzhaus-Bewegung in den 1970er und 1980er Jahren neben der Unterhaltung auch zum subtilen Ausdruck einer kritischen Haltung gegenüber der sozialistischen Ideologie gedient habe, stehe heute allein das Vergnügen am Tanzen und Musizieren im Vordergrund. Dass Volksmusik und Volkstanz heute im ungarischen Bildungssystem integriert sind, sei vor allem dem Engagement der ersten Tanzhaus-Akteure zu verdanken, welche im Zuge der Institutionalisierung der Tanzhaus-Bewegung teilweise selbst eine professionelle Laufbahn eingeschlagen haben. So hätten viele Amateur-Musiker angesehene und gefragte Berufe wie Künstler, Lehrer, Tänzer, Journalisten sowie Ethnologen und Musikwissenschaftler angenommen. Laut Ronström schließt sich mit dieser Institutionalisierung und Professionalisierung der Kreis der Tanzhaus-Bewegung. Indem sie sich durch Anpassung an die Bedürfnisse ihrer Anhänger, der Festival-Veranstalter sowie der Musik-Produzenten zu einem Bestandteil der Massenkultur entwickelt hat, wurde erneut das geschaffen, was sie anfangs kritisiert hat: eine bühnen- und massentaugliche, vereinfachte Version von Volkskultur. Nur sei diesmal nicht der Staat der einflussreichste Akteur, sondern die Anhänger der Tanzhaus-Bewegung selbst, welche die Bewegung innerhalb der Kulturindustrie lenken (Ronström, 1998, 6-7). 
Zwar wirkt Ronströms Behauptung recht pauschalisiert, jedoch gibt es zahlreiche Beispiele dafür, dass einige Gründer der Bewegung im Laufe der Jahre ein Arbeitsverhältnis mit Volkskultur-Institutionen eingangen sind oder selbst entsprechende Organisationen ins Leben gerufen haben. Dies wird mit einem Blick auf das Hungarian Heritage House veranschaulicht: Ferenc Sebő ist der technische Leiter des vom Ungarischen Kulturministerium geförderten Instituts, welches drei Abteilungen vereint: das „Ungarische Staatliche Folkloreensemble“, welches von 1981 bis 1996 von Sándor Tímár geführt wurde, das von Béla Halmos geleitete Volkskultur-Archiv sowie eine Abteilung für Volkskunst.

Aus den bisher erläuterten Aspekten lässt sich schlussfolgern, dass der oppositionelle Charakter der Tanzhaus-Bewegung durch deren Etablierung als akzeptiertes Kulturevent deutlich in den Hintergrund gerückt wurde. Jedoch ist nicht eindeutig, ob dies das Ziel der ungarischen Kulturpolitik war oder ob auch die Anhänger selbst eine derartige Entwicklung angestrebt haben. Jedenfalls zog dieser Wandel sowohl positive als auch negative Konsequenzen für die Akteure der Tanzhaus-Bewegung nach sich: Die Tanzhaus-Musiker hatten durch die staatliche Unterstützung die Möglichkeit, ihre Leidenschaft zur Profession zu machen und nationale wie internationale Anerkennung zu erlangen. Dafür mussten sie sich jedoch an die offiziellen Vorgaben der Arbeit- und Auftraggeber halten, was eine Einschränkung ihres selbstständigen musikalischen Schaffens darstellte. Gleichzeitig gewann das Tanzhaus an Popularität, sodass es auch heute noch eine bedeutende Rolle spielt - allerdings nicht mehr als Gegenkultur mit Schwerpunkt auf Volksmusik und Volkstanz, sondern als Kulturevent der Unterhaltungsszene, welches nicht nur von ungarischen Jugendlichen, sondern auch von Touristen gern besucht wird. Das heutige Milieu der Tanzhaus-Enthusiasten wird zutreffender als „Tanzhaus-Szene“ bezeichnet, da der Begriff „Tanzhaus-Bewegung“ ein politisches Potential suggeriert, welches nicht mehr vorhanden ist.

\subsection{Die Rolle der heutigen Tanzhaus-Szene}

\section{Das neue Format des Tanzhauses}

Die heutige Musiklandschaft hat sich gegenüber derjenigen der 1970er Jahre bedeutend verändert. Es haben sich unzählige Musikgenres herausgebildet, die mittels der weltweit zugänglichen Massenmedien verbreitet wurden. Besonders durch das Experimentieren mit neuen oder neu entdeckten Musikstilen und Musikinstrumenten konnte das vorhandene Repertoire ständig erweitert werden. Zudem wurde durch den technischen Fortschritt eine Allgegenwärtigkeit und Ver- 
fügbarkeit von Musik ermöglicht, welche in diesem Maße vor 40 Jahren nicht vorhanden war. Dies kann bedeuten, dass das Interesse für typische, regionale Volksmusik durch das Streben nach neuen musikalischen Einflüssen aus aller Welt verdrängt wird. Denoch finden Tanzhaus-Veranstaltungen im ungarischen Kulturangebot nach wie vor ihren Platz. Dies lässt sich auf verschiedene Art und Weise erklären: Erstens bietet auch die heutige Tanzhaus-Szene genügend Raum für musikalische Experimente, schließlich war und ist sie durch Merkmale wie Kreativität, Improvisation und Spontaneität geprägt. Zweitens findet als Gegenpol zum vorherrschenden Innovationsstreben eine Rückbesinnung auf traditionelle Musik und Tänze statt, welche bei den verschiedenen Tanzhaus-Veranstaltungen ausgelebt werden kann. Drittens hat sich durch entsprechende kulturindustrielle Prozesse mittlerweile eine vielseitige Tanzhaus-Szene herausgebildet. Ihr Angebot reicht von Tanzhaus-Abenden, wie sie in den Klubs der 1970er und 1980er Jahre stattgefunden haben, über Vereinigungen wie die „Dance-House Guild“ (Táncház Egyesület) bis hin zu internationalen Tanzhaus-Festivals.

Die „Dance-House Guild“ wurde 1990 gegründet, um ein nationsweites Netzwerk der Tanzhaus-Bewegung zu schaffen, professionelle Events und Festivals zu organisieren sowie Kooperationen mit verschiedenen ungarischen Institutionen aufzubauen. Dementsprechend vereint sie sowohl Künstler und Ethnologen als auch Amateure von Volksmusik und Volkstanz, welche durch die eigens herausgegebene Zeitschrift „folkMAGazin“ über aktuelle Ereignisse und Entwicklungen in der Tanzhaus-Szene informiert werden. Die Tatsache, dass sie eine erfolgsversprechende, wirtschaftliche Verbindung von Tanzhaus und verwandten Kulturbranchen wie Volkskunst und traditionelles Handwerk bieten möchte, verweist jedoch auf die folkloristische Orientierung der Organisation. Wie aus der Internetpräsenz der „Dance-House Guild“ hervorgeht, existieren derzeit mehr als 50 Musikgruppen, welche in Ungarn und im Ausland regelmäßig Tanzhaus-Abende gestalten und sich neben den Auftritten auch über den Verkauf von CDs, Videos und Büchern finanzieren (Dance-House Guild, 2009).

Wie anhand der oben aufgeführten Punkte verdeutlich wurde, hat die Tanzhaus-Bewegung ein neues Format angenommen, mit dem die gegenwärtigen Ansprüche an Unterhaltung in Verbindung mit Volkskultur befriedigt werden können. Dementsprechend hat sich die ehemals als Gegenkultur mit oppositionellem Charakter bekannte Tanzhaus-Bewegung zu einer akzeptierten, erfolgreichen Tanzhaus-Szene entwickelt. Ihre Popularität lässt sich bereits mit einem Blick in das ungarische Online-Kulturprogramm „Est“ feststellen: Fast jeden Tag findet in einer Kneipe oder einem Musik- und Kulturzentrum wie dem „Fonó“, dem „Budapest Jazz Club“ oder dem „Gödör Klub“ eine Tanzhaus-Veranstaltung statt (Est, 2009). 


\section{Volkskultur in der Gegenwart}

In dem bereits beschriebenen neuen und sich weiter entwickelnden Format des Tanzhauses bleiben traditionelle Volkstänze und Volksmusik unter den Jugendlichen präsent. Dániel Lipták, ein junger Volksmusiker und Student aus Szeged, hebt in einem Interview das besondere Prinzip der Tanzhaus-Bewegung hervor, welches aus der einzigartigen Mischung von Tradition und Innovation besteht: Einerseits lassen sich die Musiker durch die aus verschiedenen Regionen stammenden Lieder und Tänze inspirieren, welche sie durch die Teilnahme an Tanzhaus- und Volksmusik-Camps kennen lernen, wo sie den „Dorfmusikern“ begegnen und von ihnen unterrichtet werden. Andererseits haben die Tanzhaus-Musiker genügend Spielraum für eine innovative Gestaltung des Programms, was durch das Element der Improvisation und das Experimentieren mit beispielsweise elektronischen Instrumenten gewährleistet ist. Außerdem bringen die Musiker stets persönliche Gedanken und Gefühle mit in die Musik ein, was ebenfalls für die Gegenwärtigkeit der Veranstaltungen bedeutsam ist (Hevesi, 2007).

Ausgehend von der Tatsache, dass gegenwärtig allein in Budapest fast täglich diverse Tanzhaus-Veranstaltungen angeboten werden und über das Jahr verteilt mehrere größere Tanzhaus-Treffen stattfinden, lässt sich schlussfolgern, dass bestimmte Elemente der Volkskultur, vor allem Musik und Tanz, auch heute noch präsent sind. Besonders hervorzuheben ist schließlich der Aspekt der ,gelebten Tradition", welcher sich am Beispiel der Tanzhaus-Bewegung veranschaulichen lässt. Heute wie seit Beginn der Bewegung vor 40 Jahren werden die bei den Tanzhaus-Veranstaltungen praktizierten traditionellen Elemente nicht passiv wahrgenommen, sondern durch aktive Partizipation angewendet und fortgeführt. Dies ist ein Hinweis darauf, dass Volkskultur weiterhin einen Teil der Gesellschaft darstellt, welche sich in einem historischen Prozess befindet, und ihre Funktion als Unterhaltungsform beständig ausübt. Dabei ist zu betonen, dass Volkskultur in diesem Sinne nicht als Volkskultur per se zu verstehen ist, sondern als Retrokultur. Dies bedeutet, dass Volkskultur in der Gegenwart, im Gegensatz zu ihrer ursprünglichen Form, nicht nur im bäuerlichen Umfeld, sondern vor allem auch von städtischen Intellektuellen geschätzt und ausgeübt wird. So wird an einem Tanzhaus-Abend die als bäuerlich kategorisierte Volkskultur im urbanen Raum wieder lebendig.

\section{Interkulturelle Begegnung}

Bei einer näheren Betrachtung der heutigen Tanzhaus-Szene ist auffällig, dass die Musik und die Tänze aus verschiedenen Ländern stammen: Es gibt regelmäßig moldawische, griechische und slowakische Tanzhaus-Abende und das jähr- 
lich stattfindende Ungarische Nationale Tanzhaus-Festival wirbt mit Volksmusik sowie Volkstänzen aus dem Karpatenbecken, welches durch seine ethnische Vielfalt charakterisiert ist. Der Zusammenhang zwischen den verschiedenen Herkunftsländern der bei den Tanzhaus-Veranstaltungen vorgestellten und ausgeübten Elemente der Volkskultur besteht darin, dass sie entweder den Auslandsungarn, also den außerhalb des ungarischen Staatsgebiets ansässigen Ungarn, oder den in Ungarn lebenden ethnischen Minderheiten zuzuordnen sind. Von Beginn an diente die Tanzhaus-Bewegung einerseits der Solidaritätsbekundung mit den im Ausland lebenden ungarischen Bevölkerungsgruppen und lenkte die öffentliche Aufmerksamkeit auf die seitens der ungarischen Regierung vernachlässigte Minderheitenpolitik, andererseits bot sie gleichzeitig eine Gelegenheit für die Auseinandersetzung mit der Volkskultur anderer Ethnien. Dieser Hintergrund spiegelt sich in der heutigen Tanzhaus-Szene wider, jedoch weniger im politischen Sinne als im symbolischen, sodass sie dementsprechend als Plattform für interkulturelle Begegnungen gesehen werden kann.

Da es sowohl in Ungarn als auch in Schweden, Irland und in verschiedenen osteuropäischen Ländern ähnliche Bewegungen gab, welche sich bewusst mit Volkstanz und Volksmusik auseinandersetzten, lässt sich folgende Aussage formulieren: Festivals, wie sie regelmäßig im Rahmen der ungarischen Tanzhaus-Szene stattfinden, bilden aufgrund ihrer Gemeinsamkeiten mit anderen Bewegungen zur Wiederbelebung von Volksmusik und Volkstanz eine günstige Gelegenheit zur interkulturellen Begegnung. Dabei können die unterschiedlichen Traditionen der jeweiligen Ethnien gelehrt und gelernt werden. Darüber hinaus spielt die zu überwindende Sprachbarriere zur angemessenen Verständigung eine der größten Herausforderungen im Rahmen interkultureller Begegnungen eine Nebenrolle, denn Volksmusik und Volkstanz benötigen nicht unbedingt eine Sprache als Medium. Schließlich können die entsprechenden Kenntnisse und Kompetenzen durch Zuschauen, Zuhören und Nachahmen überliefert werden ein Prinzip, welches sich bereits zu Beginn der Tanzhaus-Bewegung bewährt hat.

\section{Zusammenfassung}

An dieser Stelle wird die eingangs aufgeworfene Frage wieder aufgegriffen: Welche Rolle spielt die ungarische Tanzhaus-Bewegung für die Volkskultur in der Moderne?

Zunächst bietet sich eine Zusammenfassung der mit dieser Fragestellung einhergehenden Aspekte an, da diese für die entsprechende Beantwortung bedeutsam sind. Volkskultur ist im Zusammenhang mit der ungarischen Tanzhaus-Bewegung sowie der heutigen Szene als Retrokultur zu verstehen. Zum einen, da Volksmusik und Volkstanz durch die Tanzhaus-Anhänger neu entdeckt und in 
verschiedene Kontexte gesetzt werden, und zum anderen, da ein Tanzhaus-Abend einen Prozess der Reproduktion von Elementen der Volkskultur darstellt. Das Reservoir der Volkskultur dient praktisch als Experimentierfeld, welches neben zahlreichen Anregungen die Möglichkeit zum kreativen Umgang mit den verschiedenen Kulturgütern bietet. Diese Gelegenheit wurde und wird von den Musikern und Tänzern der Tanzhaus-Bewegung und -Szene ausgiebig genutzt, was durch die experimentellen und improvisatorischen Elemente deutlich zum Ausdruck kommt.

Das Verhältnis zwischen Volkskultur und Moderne ist durch eine wechselseitige Beziehung und Beeinflussung gekennzeichnet. Warum die Beschäftigung mit den verschiedenen Elementen von Volkskultur, in Bezug auf die Tanzhaus-Bewegung mit Volksmusik und Volkstanz, gerade in diesem Zeitalter stattfindet, lässt sich durch die Eigenschaften der Moderne begründen: Zwar stellt sie aufgrund des technischen Fortschritts, der Schnelllebigkeit und Individualität sowie der Konsumorientierung eine Herausforderung für Volkskultur dar. Jedoch können diese Merkmale der Moderne ein Bedürfnis nach Beständigkeit, Geschichtlichkeit sowie Geselligkeit hervorrufen. Diese Eigenschaften sind für Volkskultur charakteristisch, was den Rückgriff auf ihre entsprechenden Elemente erklärt. Zudem verstärkt die Moderne als vermeintliche Konkurrenz und Bedrohung von Traditionen das Gefühl, die volkskulturellen Güter schützen, pflegen und fortführen zu müssen. Dies fördert auch das Interesse an Volksmusik und Volkstanz in der Tanzhaus-Szene. Weiterhin bietet das Zeitalter der Moderne, vor allem durch die technischen Entwicklungen, unzählige Möglichkeiten zur Verbreitung von Volkskultur. So wird ein kreativer, experimenteller Umgang mit den Elementen von Volkskultur ermöglicht, was gleichzeitig auch zu ihrer Modernisierung beiträgt. Nicht zuletzt durch den Einfluss der Massenmedien konnte ein umfassendes Bewusstsein über Volkskultur geschaffen werden, wenn auch dies eine Gefahr der Verklärung in sich barg und noch immer birgt. Darüber hinaus werden die für die Tanzhaus-Szene bedeutsamen interkulturellen Begegnungen durch die im Zuge der Modernisierung entwickelten weltweiten Reisemöglichkeiten wesentlich erleichtert.

Hinsichtlich der Entwicklung des ungarischen Tanzhauses ist ein deutlicher Wandel festzustellen, welcher sich auf mehreren Ebenen vollzogen hat. Geographisch gesehen wurden regionale Elemente von Volkskultur in den Städten und später in verschiedenen Ländern verbreitet. Aus einem kulturpolitischen Blickwinkel betrachtet, fand ein Etablierungsprozess der Tanzhaus-Bewegung statt, da sie zuerst als eine traditionelle Unterhaltungsform aufkam, später als eine oppositionelle Subkultur betrachtet wurde und mittlerweile als ein Teil der Unterhaltungsszene anerkannt ist. Demzufolge wird die ehemalige Tanzhaus-Bewegung in ihrer heutigen Form treffender mit dem Begriff „Tanzhaus-Szene“ umschrieben. 
Der Aspekt der Tanzhaus-Bewegung und der heutigen Tanzhaus-Szene als Träger von Volkskultur in der Moderne kommt bei den entsprechenden Veranstaltungen zum Ausdruck. Zum einen wird mit der traditionellen Volksmusik und den Volkstänzen ein abwechslungsreicher Tanzhaus-Abend gestaltet, so wird also das vielseitige Reservoir der Volkskultur genutzt. Zum anderen ist sie in Form von Retrokultur in weiten Bevölkerungsteilen, vor allem unter jungen Stadtbewohnern, verbreitet. Volksmusik und Volkstänze werden folglich als Freizeitaktivität praktiziert. Das dafür notwendige Interesse für Volkskultur entwickelt sich dabei auch als Gegenpol zum allgemeinen Streben nach Fortschritt und Entwicklung. Vor allem junge Menschen suchen eine Alternative zu den üblichen Unterhaltungsformen und werden im Reservoir der Volkskultur fündig. Diesem wird auch angesichts der Tatsache, dass traditionelle Kulturgüter von der Moderne verdrängt zu werden scheinen, eine neue Aufmerksamkeit gewidmet.

In Bezug auf diese Zusammenfassung lautet die Antwort auf die zentrale Frage: Die Rolle der ungarischen Tanzhaus-Bewegung sowie der heutigen Tanzhaus-Szene für das Fortbestehen von Volkskultur in der Moderne besteht darin, dass sie eine neuartige Form von Volkskulturpflege darstellt. Indem bei den Tanzhaus-Veranstaltungen Volksmusik und Volkstänze aus unterschiedlichen Regionen Siebenbürgens und Ungarns praktiziert werden, üben die Teilnehmer - bewusst oder unbewusst - eine Pflege von volkskulturellen Gütern aus. Somit tragen sie auf eine selbstverständliche und ungezwungene Art und Weise dazu bei, dass verschiedene Elemente von Volkskultur gelernt und gelebt werden. Durch ein Bild von Volkskultur, welches ihre Vielseitigkeit, ihre Komplexität und Aktualität symbolisiert, bleibt sie auch in der Moderne relevant und bedeutsam. Die Besucher eines Tanzhaus-Abends haben bereits ein anderes, für das Fortbestehen von Volkskultur notwendiges Bild: Sie ist nichts Vergangenes oder Vergehendes, sondern etwas Gegenwärtiges. Volkskultur wird in der Moderne gelebt - und dies nicht nur in Ungarn, sondern überall dort, wo traditionelle Elemente wie Volksmusik und Volkstanz ganz selbstverständlich von jungen Menschen zur Unterhaltung praktiziert werden.

\section{Literaturverzeichnis}

Balázs, J. (1983) Young People's Folk Music Movement in Hungary: Revival or Failure? International Music Education, ISME Yearbook, Vol. 10, 36-45.

Balogh, B. und Fülemile, A. (2008) Cultural Alternatives, Youth and Grassroots Resistance in Socialist Hungary - The Folk Dance and Music Revival. Journal of Hungarian Studies, Vol. 22, Nr. 1-2, 41-60.

Cohen B. (2001) Hungarian Dance House - Find out what all the Knee-Slapping is about! Online unter: http://www.wherebudapest.hu/2001_03/hungarian_dance_house.html. Zugriff am 30.03.2009 
Dance-House Guild (2009) Dance-House Guild's Story. Online unter: http://www.tanchaz.hu/ start_en.html. Zugriff am 30.03.2009

Dégh, L. (1984) The Institutional Application of Folklore in Hungary. Journal of Hungarian Studies, Vol. 6, Nr. 2, 195-216.

Diószegi, L. (2008) Historic Moments of Hungarian Folk Dance: From Gyöngyösbokréta to the Dance House Movement. Journal of Hungarian Studies, Vol. 22, Nr. 1-2, 3-8.

Est (2009) EST.hu - Zene, koncert, buli. Online unter: http://www.est.hu/zene. Zugriff am 30.03.2009

Frigyesi, J. (1996) The Aesthetic of the Hungarian Revival Movement. In Mark Slobin (ed.) Retuning Culture - Musical Changes in Central and Eastern Europe (Durham), 54-75.

Hevesi, M. (2007) Volkskunst und Tradition heute. Gespräch mit dem jungen Volksmusiker und Germanistikstudenten Dániel Lipták. Germanistisches Magazin-Studentenzeitschrift des Instituts für Germanistik an der Universität Szeged, Nr. 2. Online unter: http://www.arts.u-szeged.hu/gema/zeitung/13/kultur_volkskunst.htm. Zugriff am 30.03.2009

Hofer, T. (1992) Die Pflege der Volkskultur als Gegenstand populistischer, bürgerlicher und sozialer Diskussion in Ungarn. In Klaus Roth (Hg.) Die Volkskultur Südosteuropas in der Moderne (München). 263-77.

Kelemen, L. (2004) Hungarian Music, Gypsy Music, Folk Music. Online unter: http://csardas.org/pages.php?menuid=12. Zugriff am 30.03.2009

Klimó, A. (2006) Ungarn seit 1945 (Göttingen).

Kürti, L. (2001) The Remote Borderland: Transylvania in the Hungarian Imagination (New York).

Martin, Gy. (2001) Discovering Szék. Hungarian Heritage, Vol. 2, Nr. 1-2, 31-40.

Petzoldt, L. (2002) Folklore zwischen Globalisierung und Kommerz. Trans - Internet-Zeitschrift für Kulturwissenschaften, Nr. 12. Online unter: http://www.inst.at/trans/12Nr/petzold12.htm. Zugriff am 30.03.2009

Ronström, O. (1998) Revival in Retrospect. The Folk Music and Folk Dance Revival. Conference Paper, European Centre for Traditional Culture, Bulletin 4, 1-9.

Szőnyei, T. (2005) Kept on File. The Secret Service and the Hungarian Rock Scene, 1960-1990. Excerpted in: Hungarian Quarterly, Vol. 46, Nr. 179, 80-90. Online unter: http://www.hungarianquarterly.com/no179/10.shtml. Zugriff am 30.03.2009 